\title{
Bariatric Surgery and Risk of Urolithiasis: A Review
}

\author{
Authors: \\ Maliza Persaud, ${ }^{1}$ Satyendra Persaud, ${ }^{2}$ Chantal Gosine, ${ }^{1}$ Kristy Sadho, ${ }^{1}$ \\ Dilip Dan² \\ 1. San Fernando General Hospital, San Fernando, Trinidad and Tobago \\ 2. Division of Clinical Surgical Sciences, University of the West Indies, St. Augustine, \\ Trinidad and Tobago \\ *Correspondence to satyendrapersaud@yahoo.com \\ Disclosure: $\quad$ The authors have declared no conflicts of interest. \\ Received: $\quad 22.08 .21$ \\ Accepted: $\quad 12.01 .22$ \\ Keywords: $\quad$ Bariatric, endourology, sleeve, stones, urolithiasis. \\ Citation: $\quad$ EMJ Urol. 2022; DOI/10.33590/emjurology/21-00184.
}

\begin{abstract}
Obesity is a global epidemic for which dietary and lifestyle modifications alone are ineffective treatment strategies. Subsequently, more patients are opting for bariatric surgery, which has better success rates in weight loss and improvement of obesity-related comorbidities. These procedures involve anatomic alterations of the gastrointestinal tract resulting in either restriction of intake or malabsorption of nutrients. While obesity itself is an independent risk factor for urolithiasis, bariatric surgery may also adversely affect stone risk. Restrictive procedures appear to have the lowest risk, whereas malabsorptive procedures are associated with the highest risks of stone formation. Stone prevention strategies including dietary manipulation are critical in the management of the patients who have had bariatric surgery.
\end{abstract}

\section{INTRODUCTION}

Obesity (BMI: $>30 \mathrm{~kg} / \mathrm{m}^{2}$ ) has reached epidemic proportions, both globally as well as locally in the Caribbean region, without discrimination against age, sex, race, socioeconomic, and educational levels., ${ }^{1,2}$ More than one-third of the population in the USA and about one-half in Trinidad and Tobago currently meet the definition of overweight or obese..$^{3-5}$

Conservative methods such as diet and lifestyle modifications are often unsuccessful, or, at best, temporary with regards to sustainable weight loss. $^{3}$ The National Institutes of Health $(\mathrm{NIH})$ consensus panel has established guidelines regarding which patients should be considered for bariatric surgery. ${ }^{6}$ These recommendations include patients with BMI $>40 \mathrm{~kg} / \mathrm{m}^{2}$ or those with a BMI $>35 \mathrm{~kg} / \mathrm{m}^{2}$, with severe obesityassociated comorbidities. ${ }^{6,7}$ Consequently, more patients are resorting to surgical options that have demonstrated superior success rates, not only in weight loss but also in the improvement of obesity-related comorbidities. ${ }^{8}$ These reported successes have led to a six-fold increase in bariatric procedures in the USA alone over the past 10 years., ${ }^{9,10}$ However, as these procedures become more popular, various ensuing metabolic derangements appear to increase the risk of urolithiasis. ${ }^{3}$ The following discussion aims to highlight the delicate balance between the adverse effects of obesity and its treatment in the stone forming patient. 


\section{METHODOLOGY}

A PubMed literature search on obesity, bariatric procedures, and risk of nephrolithiasis was completed and all relevant studies were selected and reviewed. Key search words included all variations of obesity, kidney stones, nephrolithiasis, restrictive bariatric procedures, malabsorptive bariatric procedures, laparoscopic adjustable gastric banding (LAGB), sleeve gastrectomy (SG), jejunoileal bypass (JIB), Roux-en-Y gastric bypass (RYGB), biliopancreatic diversion and duodenal switch (BPD-DS), hyperoxaluria, and hypocitraturia. Only articles written in English were selected. Review articles, original articles, case series, and international disease statistical databases were selected; however, news articles and commentaries were excluded. Institutional review board approval was not necessary as this is a review article and does not include any patient specific details.

\section{BARIATRIC PROCEDURES}

The bariatric era commenced in the early 1950s, when it was observed that sustained weight loss can be achieved by a surgically shortened small bowel with resultant secondary malabsorption." Anatomical alterations of the gastrointestinal tract, either to restrict the quantity of food intake and/or to decrease the length of bowel through which absorption occurs, constitute the basis of bariatric surgery. ${ }^{3}$ The techniques that are currently employed in weight loss surgery, based on these anatomical modifications, are classified as restrictive, malabsorptive or a combination of the two. ${ }^{12}$

\section{Restrictive Procedures}

Restrictive procedures include the gastric balloon, LAGB, and laparoscopic SG (LSG), with the latter being the most commonly performed restrictive procedure in recent times. ${ }^{3}$ The gastric balloon is a relatively new short-term tool used in the fight against obesity and is primarily considered as a bridge to another procedure. ${ }^{13}$ Laparoscopic adjustable gastric banding requires the placement of an inflatable silicone band around the proximal portion of the stomach thereby creating a gastric pouch of about 5-15 mL in capacity. ${ }^{3}$ However, this procedure has lost popularity as it is less effective in achieving and sustaining weight loss compared with other bariatric procedures. ${ }^{14}$ Nonetheless, LAGB is advantageous in several ways, including short operating time, minor changes to the gastrointestinal tract, and the opportunity to customise the degree of restriction based on individual needs. ${ }^{15,16}$

A SG involves removing approximately $80 \%$ of the stomach, with the remaining 'sleeve' extending from the gastro-oesophageal junction

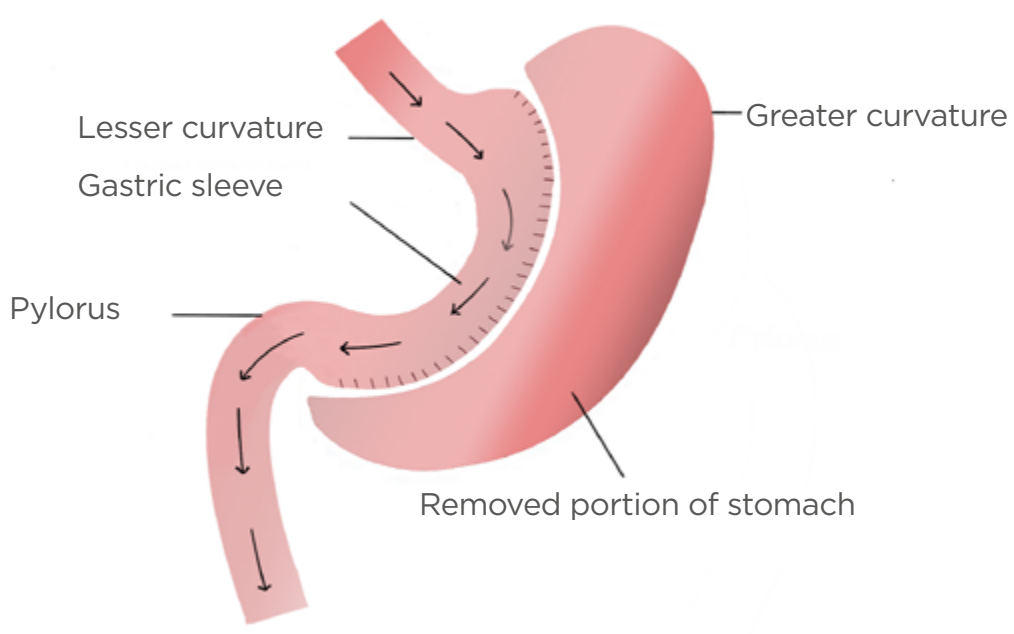

Figure 1: Vertical sleeve gastrectomy. 
to the pylorus (Figure 1). ${ }^{16}$ This procedure is associated with decreased production of the hormone ghrelin, which diminishes hunger and increases satiety. ${ }^{17}$ LSG may serve as a first stage procedure for patients who may eventually require conversion to a RYGB, a procedure that may initially be impractical due to severe obesity. ${ }^{3}$ However, over the past decade, the LSG has been considered a stand-alone operation for morbid obesity due to its encouraging early and midterm outcomes. ${ }^{18,19}$ Additionally, this procedure appears to be favoured by many bariatric surgeons due its technical simplicity and modest learning curve. ${ }^{19}$

\section{Malabsorptive Procedures}

Malabsorptive procedures achieve weight loss by decreasing the length of bowel exposed to food, thereby reducing the absorption of nutrients. ${ }^{16}$ The JIB procedure was one of the first techniques pioneered with resultant severe malabsorption of both macro- and micronutrients. ${ }^{20-22}$ However, due to life-threatening complications such as renal failure, hepatic encephalopathy, and high mortality rates, this procedure has since been abandoned in favour of safer alternatives. ${ }^{20-22}$

\section{Combined Procedures}

The evolution of malabsorptive procedures has led to combined malabsorptive and restrictive elements, with the RYGB being the most popular bariatric procedure in current times, accounting for about $80 \%$ of weight loss surgeries in the USA. ${ }^{23}$ A $20-30 \mathrm{~mL}$ gastric pouch is created by stapling the proximal stomach and separating the distal portion (Figure 2).24 The biliopancreatic limb is fashioned by dividing the small bowel about $50-150 \mathrm{~cm}$ distal to the ligament of Treitz, and a gastrojejunostomy is made between the gastric pouch and the distal separated limb of the small bowel (the Roux or alimentary limb). ${ }^{24}$ The biliopancreatic channel is anastomosed to the Roux limb $150-300 \mathrm{~cm}$ distal to the gastrojejunostomy. ${ }^{24}$ In addition to being extremely effective in achieving weight loss, altered gastric hormone secretion induced by this surgery encourages satiety and altered glucose metabolism, leading to significant improvement in the metabolic derangements of obesity. ${ }^{3}$ However, abdominal cramps and nausea may occur because of 'dumping syndrome' as undigested food enters the small bowel. ${ }^{25}$ This phenomenon may provide some therapeutic benefit as it may contribute to added weight loss by negative reinforcement of high calorie consumption. ${ }^{3,25}$

The BPD-DS, originally described by Scopinaro in 1979, also utilises a combination of malabsorptive and restrictive mechanisms (Figure 3)..$^{26,27}$ it has documented efficacy in achieving and maintaining substantial weight loss in the population who are superobese (BMI: $>50 \mathrm{~kg} /$ $\mathrm{m}^{2}$ ), with a randomised study demonstrating a $44.8 \%$ estimated body weight loss with BPD-DS

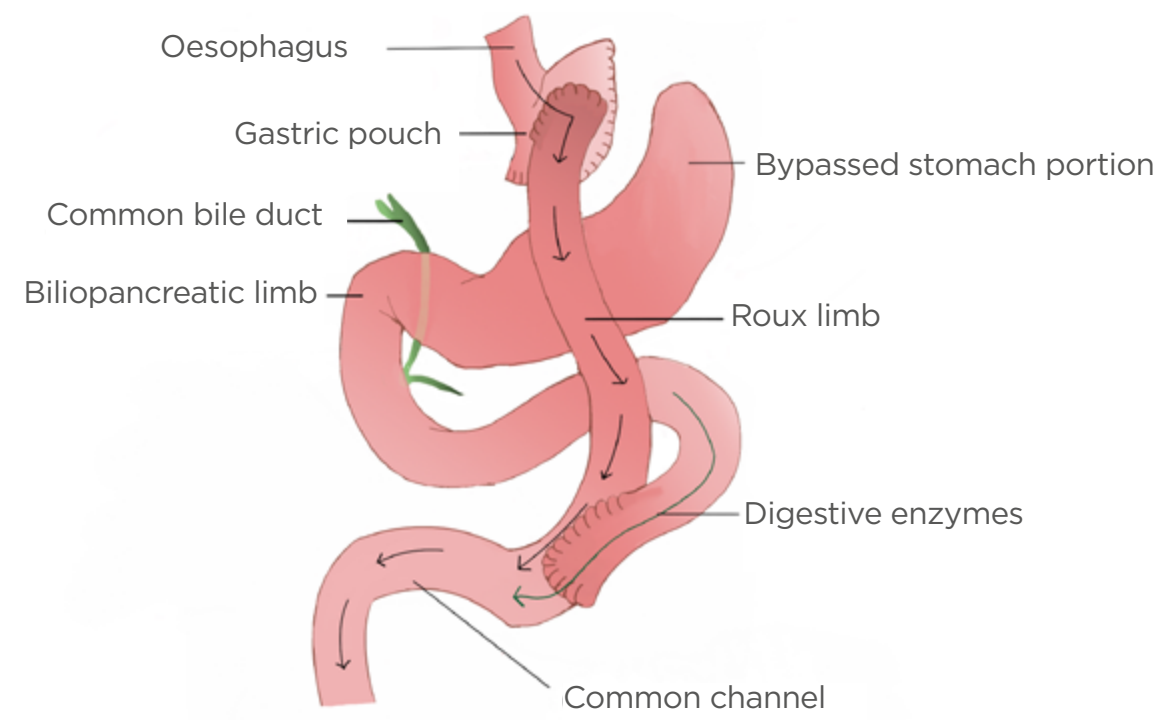

Figure 2: Roux-en-Y gastric bypass. 


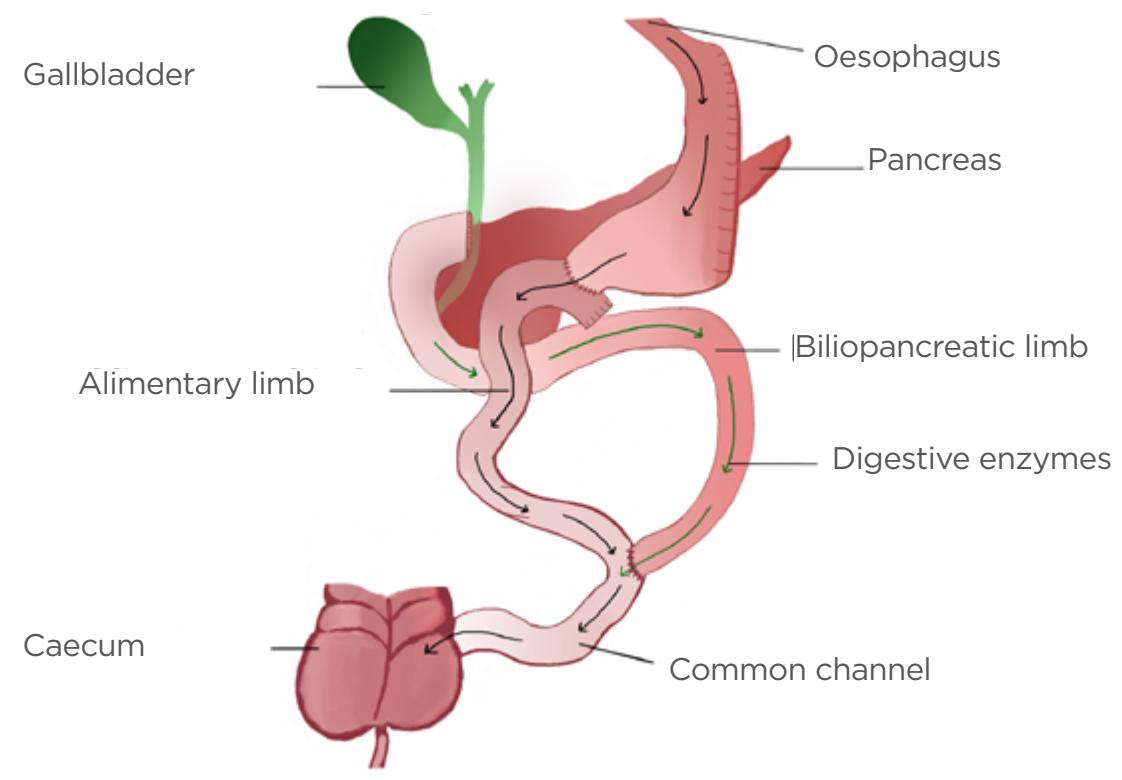

Figure 3: Biliopancreatic diversion.

compared with $31.2 \%$ following RYGB at 2 years follow-up. ${ }^{28,29}$ The dumping syndrome is avoided in this pylorus-sparing procedure and, although weight loss efficacy is better than with RYGB, there is an increased risk of malnutrition related complications. $^{3}$

\section{Comparison of Restrictive and Malabsorptive Procedures}

The urinary biochemical abnormalities seen in most patients who have had bariatric surgery are associated with an increased proclivity for urolithiasis. The exception is gastric banding, as shown in a study by Semins et al. ${ }^{30}$ where, after 2 years of follow-up of their cohort, there was no increase in the risk of developing a stone or having a surgical intervention. The investigators postulated that the possible reason for this observation might be the significant weight loss experienced by the patients post-operatively, thereby reducing their risk for urolithiasis, since obesity by itself is an independent risk factor. ${ }^{30}$ They also admitted that a follow-up of longer than 2 years may be warranted to further elucidate the relationship between gastric banding and stone risk. ${ }^{30}$

With respect to malabsorptive procedures, long-term follow-up revealed that up to $39 \%$ of patients post-JIB were found to have stones while those post-RYGB had doubled their risk of stone events. ${ }^{3}$ In a retrospective study of patients undergoing RYGB, stone prevalence was noted to be increased by $70 \%$, with $3.2 \%$ and $31.4 \%$ of post-operative patients developing de novo and recurrent stones, respectively. ${ }^{31}$ These data clearly demonstrate the increased stone risk imposed by bariatric surgery for known stone forming patients, with resultant recommendations for post-operative evaluation for metabolic abnormalities and urolithiasis. ${ }^{31}$

\section{OBESITY AND RISK OF NEPHROLITHIASIS}

The association between increased BMI and risk of stone disease has been firmly established by various epidemiological studies, with patients who are obese having as much as a two-fold increased risk. ${ }^{23,32}$ Obesity is associated with several biochemical changes to the urinary milieu that pre-dispose to urolithiasis, including a lower urinary $\mathrm{pH}$ and increased urinary excretion of calcium, oxalate, and uric acid. ${ }^{33}$ Additionally, urinary citrate, which is an important stone inhibitor, is found to be decreased in patients who are obese, a likely result of metabolic acidosis, which further potentiates the stone forming effect. ${ }^{34}$ Metabolic syndrome, which collectively encompasses obesity, dyslipidaemia, insulin resistance, and hypertension, has also been 
linked to an increased risk of nephrolithiasis. ${ }^{35,36}$ The plausible explanations include dietary factors, oxidative stress, inflammation, and insulin resistance, all of which may contribute to low urinary $\mathrm{pH}$ and subsequent development of uric acid stones. ${ }^{35}$

Dietary indulgences that are common among the population that is obese such as sweetened beverages, including soda and punches, which are associated with higher stone-forming rates. ${ }^{37}$ On the other hand, a Dietary Approaches to Stop Hypertension (DASH)-style diet has been shown to decrease kidney stone incidence due to the high vegetable and fruit, moderate low-fat dairy, and low animal protein intake. ${ }^{37}$ These favourable effects seen with the DASH diet are a result of increases in urine volume, $\mathrm{pH}$, and urinary excretion of citrate, potassium, magnesium, sulphate, and phosphate. ${ }^{33}$ Another potential pathogenic mechanism for stone formation in patients who are obese is increased intestinal absorption of oxalates resulting from a reduction in intestinal colonisation by the oxalate-degrading microorganism, Oxalobacter formigenes. ${ }^{38}$

\section{BARIATRIC PROCEDURES AND RISK OF NEPHROLITHIASIS}

Although obesity-related complications may be reversed by bariatric surgery, the evidence is mounting that these interventions may also adversely impact stone risk. $^{3}$ Each type of surgery is accompanied by varying levels of stone risk (Table 1), with restrictive procedures associated with the least risks approaching that of nonoperative controls, RYGB intermediate risk (7.65-13\%) and malabsorptive with the greatest risk (22.0-28.7\%). ${ }^{39,40,42-44}$ There are various complex underlying pathophysiologic mechanisms associated with nephrolithiasis following bariatric surgery, including low urine volume, aciduria, hyperoxaluria, and hypocitraturia. ${ }^{42,45}$ Various studies have demonstrated that malabsorptive procedures have well documented urinary metabolic derangements, with some types of surgeries such as JIB and RYGB resulting in hyperoxaluria in about $50 \%$ of patients. Conversely, restrictive procedures such as gastric banding do not appear to have this problem. ${ }^{39}$

\section{Urine Volume and $\mathrm{pH}$}

A decreased urine volume is common following bariatric procedures due to restricted gastric volumes and is one of the chief causes of urinary crystallisation and stone formation. ${ }^{42}$ Many studies have consistently documented a reduction in 24-hour urine volume in bariatric surgery patients from pre-surgery to postsurgery, with the majority of patients maintaining persistently low urine volumes thereafter. ${ }^{42}$ Acidic urine $(\mathrm{pH}:<4.6)$ has been demonstrated in several series of patients following RYGB, leading to supersaturation of uric acid and formation of uric acid stones. ${ }^{42,45}$ However, even in the presence of aciduria, the occurrence of uric acid stones is still less frequent than calcium oxalate stones following bariatric surgery. ${ }^{42}$

\section{Hyperoxaluria}

The increased incidence of urolithiasis following bariatric surgery is probably multifactorial, with the likely culprits being fat malabsorption, increased oxalate absorption and altered gut microflora. ${ }^{46}$ Fat malabsorption, which occurs

Table 1: Risk of urolithiasis following bariatric surgery. , $39,43,44^{3}$

\begin{tabular}{|l|l|}
\hline Type of procedure & Associated risk of urolithiasis \\
\hline Obese non-operative controls & $5-7 \%$ \\
\hline Restrictive (LAGB, LSG) & Low $(1.3-1.5 \%)$ \\
\hline RYGB & Intermediate (7.65-13.00\%) \\
\hline Malabsorptive procedures (JIB) & High (22.0-28.7\%) \\
\hline
\end{tabular}

JIB: jejunoileal bypass; LAGB: laparoscopic adjustable gastric banding; LSG: laparoscopic sleeve gastrectomy; RYGB: Roux-en-Y gastric bypass. 
predominantly in malabsorptive procedures, leads to enteric hyperoxaluria as fatty acids undergo saponification with intestinal calcium. This leaves behind increased intestinal quantities of unbound oxalates with subsequent colonic absorption and secretion into the urine. ${ }^{47,48}$ In a study by Nelson et al., ${ }^{49} 21$ of their 23 patients presented with a symptomatic stone resulting from enteric hyperoxaluria following RYGB. ${ }^{48}$ Furthermore, several studies have implied that patients undergoing RYGB are at higher risk for nephrolithiasis post-surgery than pre-surgery. ${ }^{26,50,51-53}$ An even greater concern was the occurrence of oxalate nephropathy and renal failure in a small subset of patients, an additional consideration for those with high urinary oxalate excretion. ${ }^{49}$

Moreover, malabsorptive bariatric procedures are associated with alterations in gut microbial flora, particularly decreased colonisation with O. formigenes, an anaerobic commensal species of bacteria present in the human colon. ${ }^{54}$ These organisms utilise oxalates as their sole energy source and decreased colonisation results in augmented oxalate absorption, hyperoxaluria, and recurrent calcium oxalate stone disease. ${ }^{42,54}$ Conversely, while restrictive bariatric procedures may provoke specific metabolic derangements, they are not associated with hyperoxaluria since a malabsorptive state is not induced. ${ }^{3}$ However, there may still be a negative impact on stone risk as a consequence of a restricted gastric volume and an overall decreased oral intake of fluids, calcium, magnesium, and citrate containing foods. ${ }^{3}$

\section{Hypocitraturia}

Citrate, a critical inhibitor of crystallisation, forms soluble complexes with calcium in the renal tubules, thereby reducing urinary supersaturation and subsequently, calcium oxalate and calcium phosphate precipitation. ${ }^{42}$ Hypocitraturia, defined as urinary citrate levels $<320 \mathrm{mg} /$ day, is associated with an increased risk of urolithiasis. This metabolic derangement is commonly observed in acidotic states as renal citrate reabsorption increases and urinary citrate excretion is reduced. ${ }^{45,55}$ Penniston et al. ${ }^{48}$ after evaluating post-operative 24-hour urine specimens, have demonstrated that patients who have had RYGB have higher urinary oxalate and lower urinary citrate levels when compared to gastric banding patients. ${ }^{8}$ While urine volume was low in both groups, it was postulated that the observed hypocitraturia in the patients who have had RYGB was a result of increased bicarbonate losses from the gastrointestinal tract. ${ }^{48}$

\section{MANAGEMENT OF STONE RISK IN PATIENTS OF POST-BARIATRIC SURGERY}

While available data indicate that bariatric procedures are associated with an increased stone forming risk, the presence of hyperoxaluria or previous stone disease are not contraindications for bariatric surgery. ${ }^{3}$ Extensive pre-operative patient counselling is, therefore, prudent, in addition to 24-hour urine evaluations prior to RYGB, especially in the setting of known stone disease. ${ }^{3}$ Additionally, monitoring of renal function, renal tract imaging, and appropriate dietary advice should be encouraged in the follow-up of these patients. ${ }^{56}$

Stone prevention therapy in patients of bariatric surgery is critical, with dietary modifications being the most important component of this strategy. ${ }^{3}$ Maintenance of daily urine output of $>2$ L/day is essential and fluid intake in the form of frequent small quantities is advisable, as some procedures may be prohibitive of large volume intake.,23 Other key elements of dietary modification include decreased oxalate ( $<100 \mathrm{mg} /$ day) and fat intake to minimise enteric absorption of oxalates. ${ }^{55}$ Elevated levels of fatty acids reaching the distal intestines due to surgical redirecting of food in patients of bariatric surgery will chelate calcium, enabling the absorption of free oxalate and resultant hyperoxaluria. ${ }^{56}$ Reduced sodium and animal protein intake are advised. Additionally, supplementation of vitamin D and calcium should be considered, with the recommended daily calcium intake of 1,000-1,200 mg/day being instituted early in the post-operative period. ${ }^{57}$ Supplemental calcium binds and enhances oxalate excretion, so its intake should be timed to correspond with oxalate consumption. Oral citrate salts such as calcium or potassium citrate can be used to correct metabolic acidosis and hypocitraturia. ${ }^{55}$

The oral administration of 0 . formigenes or its oxalate degrading enzymes is being investigated in patients with primary hyperoxaluria with the 
aim of increasing colonic oxalate metabolism, thereby decreasing systemic absorption. ${ }^{26}$ While further studies regarding this strategy are required, this option may also prove useful in patients with bariatric hyperoxaluria. ${ }^{3}$

\section{CONCLUSION}

Obesity is a well-known risk factor for urolithiasis but, on the other hand, its management by some forms of bariatric surgery is associated with an exacerbation of this risk. However, there is a definite role for bariatric surgical interventions in the management of obesity and its multitude of related comorbidities. Current available literature appears to concur that a purely restrictive procedure such as gastric banding is associated with a much lower stone forming rate than malabsorptive procedures. The biochemical changes induced by bariatric surgery, particularly hyperoxaluria and hypocitraturia, are intricately linked to nephrolithiasis. It is hoped that a better understanding of their underlying pathophysiology may help to prevent or modify the risk of kidney stones in this population. Fortunately, the multifactorial nature of bariatric surgery-associated lithogenicity lends itself well to a broad spectrum of prophylactic approaches, which have demonstrable efficacy in preventing oxalate nephrolithiasis and downstream chronic renal impairment.

\section{Take Home Points}

$>$ Bariatric surgical techniques involve reconfiguration of the digestive tract to achieve weight loss by volume restriction, malabsorption, or a combination of both. Purely restrictive procedures are associated with a lower stone forming rates than malabsorptive procedures.

$>$ Obesity is associated with a lower urinary $\mathrm{pH}$ and increased excretion of oxalate, calcium, and uric acid, and decreased urinary citrate, which all predispose to urolithiasis.

$>$ Hyperoxaluria, which occurs more commonly in malabsorptive bariatric procedures, are due to fat malabsorption, increased oxalate absorption and altered gut microflora.

$>$ Hypocitraturia (urinary citrate level: $<320$ $\mathrm{mg} /$ day) frequently occurs in certain malabsorptive procedures such as RYGB.

> Bariatric procedures are not contraindicated in the presence of hyperoxaluria or previous stone disease.

> Dietary modification in patients of postbariatric surgery is critical in the prevention of stones. These include maintaining a daily urine output of $>2$ L/day; decreased oxalate, fat, sodium and animal protein intake; and supplementation of vitamin D, calcium, and oral citrate.

\section{References}

1. Deitel M. Overweight and obesity worldwide now estimated to involve 1.7 billion people. Obes Surg. 2003;13(3):329-30.

2. Dan $\mathrm{D}$ et al. Bariatric surgery in the Caribbean: is it safe in a low-volume, third world setting? Minim Invasive Surg. 2012;427803.

3. Hyams ES, et al. Bariatric surgery and risk of stone disease. AUA Update Series. 2012;31(27):273-80.

4. Government of Trinidad and Tobago. Panamerican steps chronic non-communicable disease risk factor survey final report. 2012 Available at: https://www.who. int/ncds/surveillance/steps/ TrinidadAndTobago_2011_STEPS Report.pdf. Last accessed: 17 January 2022.

5. Flegal KM et al. Prevalence of obesity and trends in the distribution of body mass index among US adults, 19992010. JAMA. 2012;307(5):491-7.

6. Gastrointestinal surgery for severe obesity: National Institutes of Health consensus development conference statement. Am J Clin Nutr. 1992;55(Suppl 2):615S-9S

7. Schneider BE, Mun EC. Surgical management of morbid obesity. Diabetes Care. 2005;28(2):475-80.

8. Sjöström L et al. Effects of bariatric surgery on mortality in Swedish obese subjects. N Engl J Med. 2007;357(8):741-52

9. Buchwald $\mathrm{H}$ et al. Bariatric surgery: a systematic review and meta-analysis. JAMA. 2004;292(14):1724-37

10. Santry HP et al. Trends in bariatric surgical procedures. JAMA. 2005;294(15):1909-17.

11. Kremen AJ et al. An experimental evaluation of the nutritional importance of proximal and distal small intestine. Ann Surg. 1954;140(3):439-48.

12. Canales BK, Gonzalez RD. Kidney stone risk following Roux-en-Y gastric bypass surgery. Transl Androl Urol. 2014;3(3):242-9.

13. Ball $W$ et al. Effectiveness of intra-gastric balloon as a bridge to definitive surgery in the super obese. Obes Surg. 2019;29(6):1932-6.

14. Wang $S$ et al. Comparison between laparoscopic sleeve gastrectomy and laparoscopic adjustable gastric banding for morbid obesity: a metaanalysis. Obes Surg. 2013;23(7):9806.

15. Chapman AE et al. Laparoscopic adjustable gastric banding in the treatment of obesity: a systematic literature review. Surgery. 


\section{4;135(3):326-51.}

16. Karmali S et al. Bariatric surgery a primer. Can Fam Physician. 2010;56(9):873-9.

17. Arias E, et al. Mid-term follow-up after sleeve gastrectomy as a final approach for morbid obesity. Obes Surg. 2009;19(5):544-8.

18. Boza C et al. Laparoscopic sleeve gastrectomy as a stand-alone procedure for morbid obesity: Report of 1,000 cases and 3-year follow-up. Obes Surg. 2012;6(22):866-71.

19. Sucandy I et al. Comparison of vertical sleeve gastrectomy versus biliopancreatic diversion. N Am J Med Sci. 2014;6(1):35-8

20. Requarth JA et al. Long-term morbidity following jejunoileal bypass: the continuing potential need for surgical reversal. Arch Surg. 1995; 130(3):318-25

21. Mechanick Jl et al. American Association of Clinical

Endocrinologists, The Obesity Society, and American Society for Metabolic \& Bariatric Surgery medical guidelines for the clinical practice for the perioperative nutritional, metabolic and nonsurgical support of the bariatric surgery patient. Surg Obes Relat Dis. 2008;4(Suppl 5):S109-84

22. Colquitt $\mathrm{J}$ et al. Surgery for morbid obesity. Cochrane Database Syst Rev. 2005;(4):CD003641.

23. Gonzalez RD, Canales BK. Kidney stone risk following modern bariatric surgery. Current Urology Reports. 2014;15(5):401.

24. Kizy S et al. Bariatric surgery: a perspective for primary care. Diabetes Spectr. 2017;30(4):265-76

25. Vavricka SR, Greuter T. Gastroparesis and dumping syndrome: current concepts and management. J Clin Med. 2019;8(8):1127.

26. Scopinaro $\mathrm{N}$ et al. Biliopancreatic bypass for obesity. II. Initial experience in man. Br J Surg. 1979;66(9):618-20.

27. Lieske JC et al. Nephrolithiasis after bariatric surgery for obesity. Semin Nephrol. 2008;15(5):401.

28. Anderson B et al. Biliopancreatic diversion: the effectiveness of duodenal switch and its limitations. Gastroenterol Res Pract. 2013;2013:974762

29. Sovik TT et al. Weight loss, cardiovascular risk factors, and quality of life after gastric bypass and duodenal switch. Ann Intern Med. 2011;155(5):281-91.

30. Semins MJ et al. The effect of gastric banding on kidney stone disease. Urology. 2009;74(4):746-9.

31. Durrani $O$ et al. Analysis of stone disease in morbidly obese patients undergoing gastric bypass surgery. J Endourol. 2006;20(10):749-52.

32. Khan SR et al. Kidney stones. Nat Rev Dis Primers. 2016;2:16008.

33. Shavit $L$ et al. Effect of being overweight on urinary metabolic risk factors for kidney stone formation. Nephrol Dial Transplant. 2015;30(4):607-13.

34. Dhar NB et al. Jejunoileal bypass reversal: effect on renal function, metabolic parameters and stone formation. J Urol. 2005;174(5):184446.

35. Rendina D et al. Metabolic syndrome and nephrolithiasis: a systematic review and meta-analysis of the scientific evidence. J Nephrol. 2014;27(4):371-6.

36. West B et al. Metabolic syndrome and self-reported history of kidney stones: The National Health and Nutrition Examination Survey (NHANES III) 1988-1994. Am J Kidney Dis. 2008;51(5):741-7

37. Taylor EN et al. DASH-style diet associates with reduced risk for kidney stones. J Am Soc Nephrol. 2009;20(10):2253-9.

38. Roswitha $\mathrm{S}$ et al. The role of Oxalobacter formigenes colonization in calcium oxalate stone disease. $J$ Urol. 2013;83(5):1144-9.

39. Lieske JC et al. Kidney stones are common after bariatric surgery. Kidney Int. 2014;87(4):839-45.

40. Bhatti UH et al. Nephrolithiasis after bariatric surgery: a review of pathophysiologic mechanisms and procedural risk. Int J Surg. 2016;36:618-23.

41. Prochaska M, Worchester E. Risk factors for kidney stone formation following bariatric surgery. Kidney360. 2020;1(12):1456-61.

42. Chen $T$ et al. The effect of restrictive bariatric surgery on urolithiasis. J Endourol. 2013;27(2):242-4.

43. Matlaga BR et al. Effect of gastric bypass surgery on kidney stone disease. J Urol. 2009;181:2573-7.
44. Gkentzis A et al. Urolithiasis in inflammatory bowel disease and bariatric surgery. World J Nephrol. 2016.6;5(6):538-46.

45. Sakhaee $\mathrm{K}$ et al. The effects of bariatric surgery on bone and nephrolithiasis. Bone. 2016;84:1-8.

46. Park AM et al. A prospective study of risk factors for nephrolithiasis after Roux-en-Y gastric bypass surgery. J Urol. 2009;182(5):2334e2339.

47. Tarplin S et al. Stone formation and management after bariatric surgery. Nat Rev Urol. 2015;12(5):263-70.

48. Yacoubian A, Nasr R. Review of post bariatric surgery effects on common genitourinary physiology. Int Braz J Urol. 2018;44(4):680-7.

49. Penniston KL et al. Gastric band placement for obesity is not associated with increased urinary risk of urolithiasis compared to bypass. J Urol. 2009;182(5):2340-2346.

50. Nelson WK et al. Enteric hyperoxaluria, nephrolithiasis, and oxalate nephropathy: potentially serious and unappreciated complications of Roux-en-Y gastric bypass. Surg Obes Relat Dis. 2005;1(5):481-5.

51. Asplin JR, Coe FL. Hyperoxaluria in kidney stone formers treated with modern bariatric surgery. J Urol. 2007;177:565-9

52. Sinha MK et al. Hyperoxaluric nephrolithiasis is a complication of Roux-en-Y gastric bypass surgery. Kidney Int. 2007;72(1):100-7.

53. Chang AR et al. Bariatric surgery and kidney-related outcomes. Kidney Int Rep. 2017;2(2):261-70.

54. Canales BK, Hatch M. Oxalobacter formigenes colonization normalizes oxalate excretion in a gastric bypass model of hyperoxaluria. Surg Obes Relat Dis. 2017:13(7):1152-7.

55. Zuckerman JM, Assimos DG. Hypocitraturia: pathophysiology and medical management. Rev Urol. 2009;11(3):134-44.

56. Espinoso-Grosso PM, Canales BK. Kidney stones after bariatric surgery: risk assessment and mitigation. Bariatr Surg Pract Patient Care. 2017;12(1):3-9.

57. Duffey BG et al. Roux-en-Y gastric bypass is associated with early increased risk factors for development of calcium oxalate nephrolithiasis. J Am Coll Surg. 2008;206(6):1145-53. 